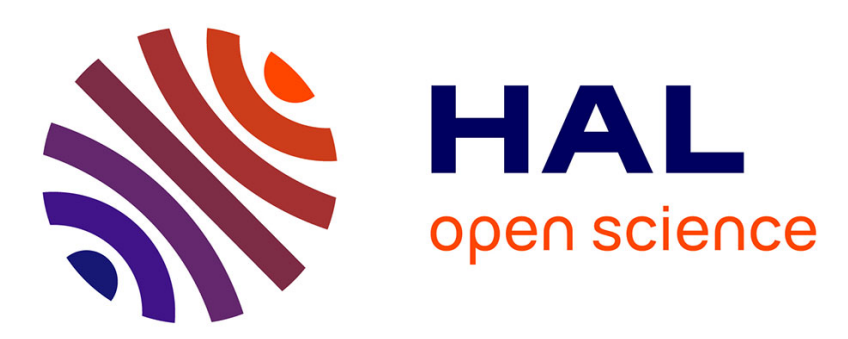

\title{
Optimisation du profil de dopage d'un MESFET réalisé par implantation ionique
}

\author{
J.L. Cazaux, Jacques Graffeuil, D. Pavlidis
}

\section{To cite this version:}

J.L. Cazaux, Jacques Graffeuil, D. Pavlidis. Optimisation du profil de dopage d'un MESFET réalisé par implantation ionique. Revue de Physique Appliquée, 1986, 21 (2), pp.139-149. 10.1051/rphysap:01986002102013900 . jpa-00245419

\section{HAL Id: jpa-00245419 https://hal.science/jpa-00245419}

Submitted on 1 Jan 1986

HAL is a multi-disciplinary open access archive for the deposit and dissemination of scientific research documents, whether they are published or not. The documents may come from teaching and research institutions in France or abroad, or from public or private research centers.
L'archive ouverte pluridisciplinaire HAL, est destinée au dépôt et à la diffusion de documents scientifiques de niveau recherche, publiés ou non, émanant des établissements d'enseignement et de recherche français ou étrangers, des laboratoires publics ou privés. 
Classification

Physics Abstracts

$72.80 \mathrm{E}-61.70 \mathrm{~W}-02.70$

\title{
Optimisation du profil de dopage d'un MESFET réalisé par implantation ionique
}

\author{
J. L. Cazaux, J. Graffeuil (*) \\ Laboratoire d'Automatique et d'Analyse des Systèmes du C.N.R.S., \\ 7, avenue du Colonel Roche, 31077 Toulouse Cedex, France
}

et D. Pavlidis

THOMSON-C.S.F./O.A.G., Domaine de Corbeville, 91401 Orsay, France

(Reçu le 8 juillet 1985, révisé le 17 octobre 1985, accepté le 22 octobre 1985)

\begin{abstract}
Résumé. - On analyse l'interdépendance entre les performances d'un transistor à effet de champ en arséniure de gallium et son profil de dopage réalisé par implantation ionique. On étudie en particulier l'influence des conditions d'implantation (énergie et dose implantées) ainsi que celle du creusement de grille (" recess ») préalablement à la réalisation de la barrière Schottky. Les résùltats théoriques ont été obtenus à l'aide d'un simulateur permettant d'accéder aux propriétés statiques et dynamiques petits signaux du transistor à effet de champ pour tout profil de dopage non uniforme. Le temps de calcul a été réduit au maximum en associant des techniques analytiques et numériques. Le fonctionnement général du logiciel ainsi que les principes du calcul de toutes les caractéristiques électriques du composant sont détaillés. Les résultats de la simulation sont comparés aux résultats expérimentaux obtenus sur des structures réalisées en faisant varier les conditions d'implantation et la profondeur du creusement de grille. Enfin, l'influence du profil de dopage sur chacun des éléments du schéma équivalent du transistor à effet de champ GaAs est discutée en vue d'une optimisation des performances micro-ondes.
\end{abstract}

\begin{abstract}
A method is proposed to investigate the influence of doping profiles on the performance of GaAs Field Effect Transistors. We consider in particular the effect of different ion implantation energies and doses, as well as, the influence of gate recess. The static and dynamic small signal characteristics of GaAs MESFETs with nonuniform doping profiles are studied by combining analytical and numerical techniques to reduce calculation time. Details of the FET analysis and computer simulation are presented. Results are compared with experimental data obtained from FETs with different implantation conditions and gate recess depths. The influence of the doping profile on the equivalent circuit elements of GaAs MESFET's is finally investigated in view of an optimization of their microwave properties.
\end{abstract}

\section{Introduction.}

Aujourd'hui, pour les MESFETs GaAs $\left({ }^{1}\right)$ les plus performants et destinés en particulier aux circuits intégrés rapides, la zone active de type $\mathbf{N}$ du transistor est réalisée par une ou plusieurs implantations ioniques (généralement d'atomes de silicium) sur un substrat semi-isolant en arséniure de gallium [1].

Cette technique de réalisation offre de nouveaux degrés de liberté pour optimiser les performances du

(*) Aussi à l'Université P. Sabatier, Toulouse, France.

( $\left.{ }^{1}\right)$ Gallium Arsenide Metal-Semiconductor Field Effect Transistor. composant, ce que ne permettait pas aussi facilement une technique plus ancienne telle que l'épitaxie en phase vapeur.

En effet, la forme du profil de dopage implanté $N_{\mathrm{D}}(y)$, dans un plan perpendiculaire à la grille (Fig. 1). peut être choisie en effectuant des implantations multiples sous des énergies variables de manière à optimiser certaines des performances du transistor.

Cet article présente donc un simulateur prenant en compte la répartition exacte $N_{\mathrm{D}}(y)$ des impuretés dopantes dans le semiconducteur afin d'en déduire tous les paramètres électriques principaux dont la connaissance est nécessaire pour la conception de transistors optimisés, voire de circuits intégrés à base de MESFETs. 


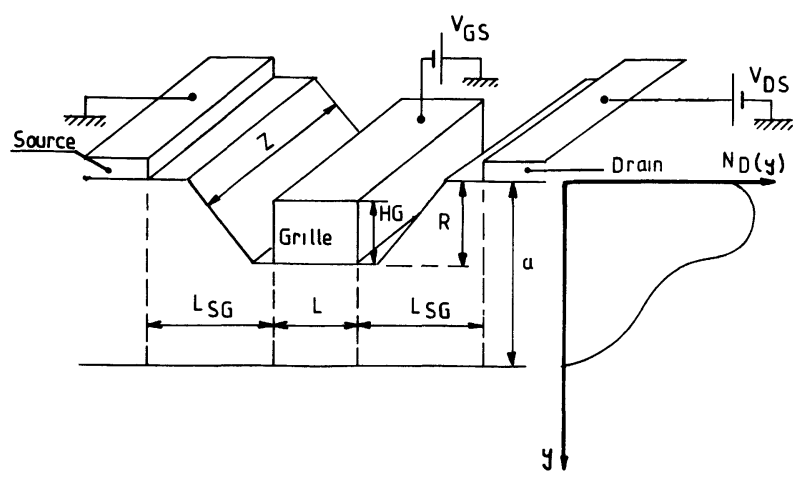

Fig. 1. - Structure et profil de dopage d'un MESFET GaAs à grille creusée.

[Cross-section and doping profile of a recessed gate $\mathrm{GaAs}$ MESFET.]

Les résultats théoriques présentés sont complétés par des résultats expérimentaux mettant en évidence l'intérêt de la simulation.

Pour contrôler la tension de pincement et minimiser les résistances d'accès $R_{\mathrm{S}}$ et $R_{\mathrm{D}}$ (Fig. 1), dont on connaît le rôle néfaste vis-à-vis des performances micro-ondes [1], les structures à grille creusée sont maintenant très répandues. On réalise sur le matériau préalablement implanté une tranchée appelée "recess » au fond de laquelle le métal de grille est déposé. Il apparaít donc que le profil final de dopage sous cette électrode dépend de la profondeur du "recess". Le simulateur que nous proposons prend en compte cette nouvelle variable.

Dans une première partie, nous décrivons le fonctionnement de ce logiciel dénommé SIMTEC. Dans la seconde, nous illustrons l'intérêt de ce simulateur dans un cas concret. Il s'agit d'étudier l'influence du profil de dopage à travers la profondeur du " recess » et les conditions d'implantation, sur les éléments du schéma électrique équivalent du transistor dont, en particulier, sa pente et ses performances en fréquence. Dans une troisième partie certains résultats de la simulation sont comparés avec des résultats expérimentaux obtenus sur des structures réalisées en faisant effectivement varier les paramètres technologiques précédents. Enfin, dans une quatrième partie, nous étudions théoriquement l'influence du profil de dopage sur tous les éléments du schéma électrique équivalent du transistor.

\section{Simulateur d'un MESFET GaAs à profil de dopage non uniforme : SIMTEC.}

2.1 LeS MODÈLES DE MESFET À PROFIL DE DOPAGE NON UNIFORME. - Divers travaux publiés à ce jour ont développé des modélisations permettant de traiter des profils de dopage quelconques. Les modèles numériques bidimensionnels doivent être écartés car ils nécessitent des temps de calcul trop longs pour une utilisation pratique, en CAO par exemple.
J. A. Higgins a présenté un modèle numérique simplifié $[2,3]$, mais qui ne permet pas de déterminer la partie réelle de l'impédance d'entrée (seule possibilité pour accéder à la fréquence maximale d'oscillation du composant).

Les travaux de J. M. Golio et R. J. Trew [4] traitent d'un modèle analytique unidimensionnel mais dont la mise en œuvre nous semble délicate en raison notamment de nombreux paramètres ajustables.

Aussi, il nous est apparu nécessaire de proposer un nouveau modèle associant des techniques analytiques et numériques. Le calcul est essentiellement unidimensionnel mais certains paramètres électriques sont déterminés par une approche bidimensionnelle. De ce fait, le simulateur proposé ici décrit le plus précisément possible le composant et autorise, grâce à son faible temps de calcul, une utilisation pour la recherche de profils de dopage optimisant les performances.

\subsection{Présentation - Paramètres d'entrée - Don-} NÉES DE SORTIE.

2.2.1 Les paramètres d'entrées nécessaires au programme sont :

i) Les dimensions géométriques de la structure étudiée (Fig. 1) telles que $Z, L, L_{\mathrm{SG}}, L_{\mathrm{GD}}$ et éventuellement $R$, profondeur du " recess ", $N_{\mathrm{K}}$, le nombre de doigts de grille, $H_{\mathrm{G}}$, l'épaisseur du métal de grille (voir Fig. 1).

ii) Certaines grandeurs électriques telles que $V_{\mathrm{bi}}$, la hauteur de barrière du contact Schottky de grille, $\rho_{\mathrm{c}}$ la résistance spécifique par unité de surface des contacts ohmiques, $\rho_{\mathrm{g}}$ la résistivité du métal de grille et $R_{\mathrm{TH}}$ (facultatif) la résistance thermique du transistor.

iii) Le profil de dopage $N_{\mathrm{D}}(y)$, variable dans le sens perpendiculaire à la surface (Fig. 1).

A partir du profil de dopage, on calcule la tension de pincement $V_{\mathrm{P}}$ du composant par [1] :

$$
V_{\mathbf{P}}=\frac{q}{\varepsilon} \int_{0}^{\infty} N_{\mathbf{D}}(y) y \mathrm{~d} y .
$$

La tension grille de seuil $V_{\mathrm{T}}$ est reliée à $V_{\mathrm{P}}$ par la relation :

$$
V_{\mathbf{T}}=V_{\mathbf{b i}}-V_{\mathbf{P}}
$$

2.2.2 Les résultats sont calculés pour un couple de tensions de polarisation du MESFET $\left(V_{G S}, V_{\text {DS }}\right)$ en régime de petits signaux. On obtient :

i) le courant $I_{\mathrm{DS}}$ qui traverse le composant du drain à la source.

ii) Les éléments du schéma équivalent de la figure 2, à savoir :

- $R_{\mathrm{S}}, R_{\mathrm{D}}, R_{\mathrm{G}}$, les résistances d'accès

- $R_{\mathrm{i}}, C_{\mathrm{i}}$, résistance et capacité d'entrée

- $g_{\mathrm{d} o}$, conductance de sortie 


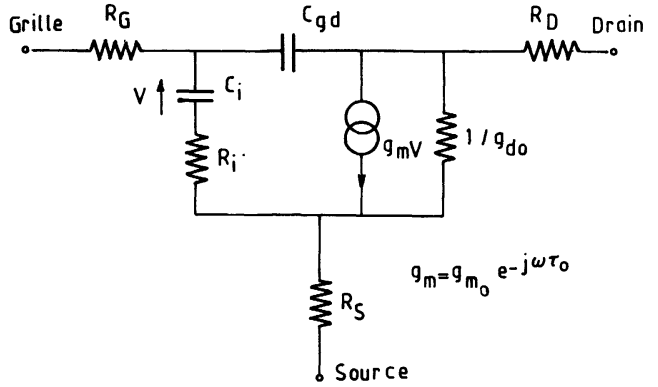

Fig. 2. - Schéma équivalent simplifié du MESFET.

[Simplified equivalent electrical network of a GaAs MESFET.] sortie

- $g_{\mathrm{m}_{0}}, C_{g_{\mathrm{d}}}$, transconductance et capacité entrée-

- $\tau_{0}$, temps de transit des porteurs sous la grille.

iii) Certaines performances micro-ondes calculées à partir de ce schéma équivalent, telles que :

- La fréquence de coupure du gain en courant $f_{\mathbf{T}}$ et la fréquence maximale d'oscillation $f_{\text {MAX }}$.

- Les paramètres $S$, le gain, le facteur de bruit approché [1], aux fréquences choisies par l'utilisateur. Pour ces dernières grandeurs, il est possible de compléter le schéma équivalent de la figure 2 par l'adjonction d'éléments parasites liés à la géométrie de la puce et à son support.

2.3 ANALYSE DU MODÈle - RELATIONS UTILISÉES. Nous utilisons les hypothèses suivantes :

- La mobilité à champ nul, $\mu_{0}$, varie avec le dopage moyen de la zone active du composant [5]. Nous proposons la relation suivante :

$$
\mu_{0}=\frac{\mu}{1+\left[\frac{N_{\mathrm{D}}}{10^{23}}\right]^{1 / 2}}
$$

où $\mu$ représente la mobilité à champ et dopage nul $\left(\mu=0,8 \mathrm{~m}^{2} \mathrm{~s}^{-1} \mathrm{~V}^{-1}\right)$ dans le GaAs [5] et $N_{\mathrm{D}}$ le dopage moyen exprimé en $\mathrm{m}^{-3}$.

- Signalons qu'il est possible de tenir compte d'un éventuel profil de mobilité variable en fonction de la profondeur $y$.

- Nous utilisons une caractéristique à deux sections pour décrire l'évolution de la vitesse en fonction du champ électrique.

$$
\begin{array}{llll}
V(E)=\frac{\mu_{0} E}{1+E / E_{0}} & \text { si } & E \leqslant E_{\mathrm{s}} \\
V(E)=V_{\text {sat }} & \text { si } & E>E_{\mathrm{s}}
\end{array}
$$

- $E_{0}$ est un paramètre phénoménologique invariable ayant les dimensions d'un champ électrique et égal à $50 \times 10^{6} \mathrm{~V} . \mathrm{m}$;
- $E_{\mathrm{s}}$ est le champ longitudinal à partir duquel les électrons atteignent leur vitesse de saturation. Il est calculé par :

$$
E_{\mathrm{s}}=\frac{V_{\text {sat }}}{\mu_{0}-\frac{V_{\text {sat }}}{E_{0}}}
$$

- $V_{\text {sat }}$ est la vitesse de dérive moyenne d'un électron, supérieure à la vitesse saturée en régime stationnaire. Afin de rendre compte de manière simplifiée des phénomènes de survitesse, nous utilisons la relation $[1,6]$ :

$$
V_{\text {sat }}=60 L^{-0,56}
$$

où $L$ est exprimée en mètres.

La transition entre les zones dépeuplées et la zone neutre du canal est dans une première version du modèle, considérée comme abrupte, c'est-à-dire que l'on pose (approximation « abrupte ») :

$$
\begin{aligned}
& n(y)=0 \quad \text { si } y<y_{\mathrm{d}} \\
& n(y)=N_{\mathrm{D}}(y) \text { si } y \geqslant y_{\mathrm{d}}
\end{aligned}
$$

où $y_{\mathrm{d}}$ est la profondeur de la zone dépeuplée avec :

$$
y_{1} \leqslant y_{\mathrm{d}} \leqslant y_{2} \quad \text { (voir Fig. 3). }
$$

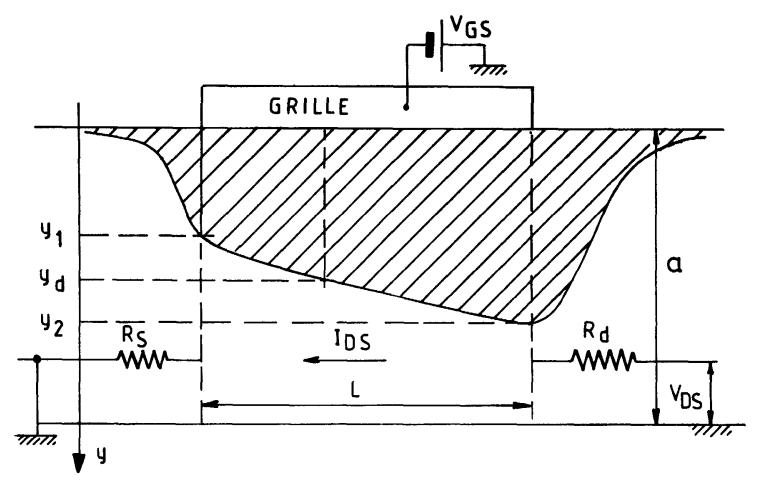

Fig. 3. - Coupe de la zone active d'un MESFET en régime ohmique montrant la région dépeuplée (en hachuré) et le canal conducteur.

[Cross-section of GaAs MESFET operated in ohmic region showing both the depleted and the conductive region.]

Si le temps de calcul n'est pas une priorité, on peut alors en résolvant en tout point les équations du semiconducteur, déterminer la répartition exacte des porteurs libres $n(y)$ qui est égale ni à zéro, ni à $N_{\mathrm{D}}(y)$ sur plusieurs distances $\lambda_{D}$ de Debye extrinsèques $\left({ }^{2}\right)$ autour de $y_{\mathrm{d}}$. Ceci permet d'obtenir des résultats beaucoup plus fins mais qui diffèrent néanmoins assez peu des résultats présentés ici [7].

( $\left.{ }^{2}\right) \lambda_{\mathrm{D}}=\left[\frac{\varepsilon k T}{q^{2} N_{\mathrm{D}}\left(y_{\mathrm{d}}\right)}\right]^{1 / 2}$. 
2.3.1 Analyse du régime statique. - i) Régime ohmique.

Lorsque le champ électrique longitudinal est en tout point inférieur à $E_{\mathrm{s}}$, le transistor fonctionne en régime ohmique. C'est notamment le cas lorsque la tension $V_{\mathrm{Ds}}$ n'est pas trop importante $\left(V_{\mathrm{Ds}}<L E_{\mathrm{s}}\right)$. La géométrie de la zone dépeuplée sous la grille est dans ce cas représentée sur la figure 3.

Ce régime est alors décrit par trois expressions obtenues à partir de l'équation de Poisson et de la loi d'Ohm :

$$
\begin{gathered}
V_{\mathrm{bi}}+R_{\mathrm{S}} I_{\mathrm{DS}}-V_{\mathrm{GS}}=\frac{q}{\varepsilon} \int_{0}^{y_{1}} y N_{\mathrm{D}}(y) \mathrm{d} y \\
V_{\mathrm{DS}}-\left(R_{\mathrm{S}}+R_{\mathrm{D}}\right) I_{\mathrm{DS}}=\frac{q}{\varepsilon} \int_{y_{1}}^{y_{2}} y N_{\mathrm{D}}(y) \mathrm{d} y \\
I_{\mathrm{DS}}\left(L+\frac{q}{\varepsilon E_{0}} \int_{y_{1}}^{y_{2}} y N_{\mathrm{D}}(y) \mathrm{d} y\right)= \\
=\frac{q^{2} Z}{\varepsilon} \int_{y_{1}}^{y_{2}}\left(\int_{y}^{a} \mu_{0}\left(y^{\prime}\right) N_{\mathrm{D}}\left(y^{\prime}\right) \mathrm{d} y^{\prime}\right) y N_{\mathrm{D}}(y) \mathrm{d} y
\end{gathered}
$$

( $a$ est l'épaisseur totale de la zone active, en pratique on prend $a \simeq 0,5 \mu \mathrm{m}$ ).

La résolution de ce système d'équations à trois inconnues $\left(y_{1}, y_{2}\right.$ et $\left.I_{\mathrm{DS}}\right)$ est réalisée numériquement et nous obtenons la valeur recherchée du courant drain-source pour tout couple $\left(V_{\mathrm{GS}}, V_{\mathrm{DS}}\right)$.

ii) Régime saturé.

Lorsque la tension $V_{\mathrm{Ds}}$ augmente, on entre dans un régime saturé où les porteurs sont supposés conserver une vitesse limite égale à $V_{\text {Sat }}$ sur une certaine fraction du parcours. On suppose alors que le canal se divise en deux régions comme indiqué sur la figure 4.

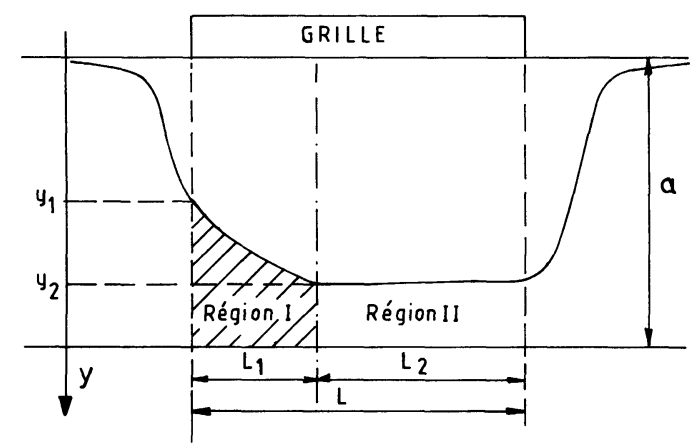

Fig. 4. - Coupe de la zone active d'un MESFET en régime saturé montrant la partition du canal conducteur en deux régions.

[Cross-section of GaAs MESFET operated in saturation showing a two region partition of the conductive channel.]

- La région I est une région où le champ électrique reste en tout point inférieur à $E_{\mathrm{S}}$. On peut donc utiliser les équations (7) et (9) en remplaçant $L$ par $L_{1}$.
- Dans la région II, les porteurs ont atteint la vitesse de saturation $V_{\text {sat }}$ et on doit écrire :

$$
I_{\mathrm{DS}}=q V_{\mathrm{sat}} Z \int_{y_{2}}^{a} N_{\mathrm{D}}(y) \mathrm{d} y .
$$

Afin d'évaluer la différence de potentiel entre les deux extrémités de la région II, nous utilisons la méthodologie proposée par Grebene et Ghandi [8] pour trouver une solution analytique à l'équation de Poisson bidimensionnelle dans cette région. Dans ce but, nous définissons une épaisseur équivalente $" a_{\mathrm{s}}$ " de la couche active dans la région II par la relation :

$$
a_{\mathrm{s}}=\frac{I_{\mathrm{DS}}}{q V_{\mathrm{sat}} Z N_{\mathrm{D}}\left(y_{2}\right)}+y_{2} .
$$

Il vient alors :

$$
\begin{aligned}
V_{\mathrm{DS}} & -\left(R_{\mathrm{S}}+R_{\mathrm{D}}\right) I_{\mathrm{DS}}= \\
& =\frac{q}{\varepsilon} \int_{y_{1}}^{y_{2}} y N_{\mathrm{D}}(y) \mathrm{d} y+\frac{2 a_{\mathrm{s}} E_{\mathrm{s}}}{\pi} \sinh \left[\frac{\pi\left(L-L_{1}\right)}{2 a_{\mathrm{S}}}\right] .
\end{aligned}
$$

Le système à cinq inconnues $\left(a_{\mathrm{S}}, y_{1}, y_{2}, L_{1}\right.$ et $\left.I_{\mathrm{DS}}\right)$ constitué par les équations (7), (9), (10), (11), (12) est résolu numériquement de la même façon que pour le régime ohmique afin d'obtenir notamment les caractéristiques de sortie courant-tension.

En annexe I, nous présentons une correction à appliquer à la valeur du courant $I_{\mathrm{DS}}$ ainsi calculé pour tenir compte éventuellement de l'échauffement du composant.

\subsubsection{Détermination des éléments du schéma équiva-} lent. - i) Les résistances d'accès $R_{\mathrm{S}}$ et $R_{\mathrm{D}}$.

On décompose $R_{\mathrm{S}}$ en deux résistances, l'une propre au contact ohmique de source $R_{\mathrm{CT}}$ et l'autre due au barreau de semiconducteur compris entre la grille et la source, $R_{\mathrm{sC}}$ (Fig. 5).

$R_{\mathrm{CT}}$ est calculé par un modèle distribué [1]. Tout calcul fait et compte tenu de la longueur importante du contact par rapport à l'épaisseur de la couche, on obtient :

$$
R_{\mathrm{CT}}=\frac{1}{Z} \sqrt{\frac{\rho_{\mathrm{c}}}{q \int_{0}^{a} N_{\mathrm{D}}(y) \mu(y) \mathrm{d} y}} .
$$

Enfin, $R_{\mathrm{SC}}$ s'exprime en fonction de la distance $L_{\mathrm{SG}}$ et de la largeur de la zone active $W(x)$ en tout point $x$ (voir Fig. 5).

$$
R_{\mathrm{SC}}=\int_{0}^{L_{\mathrm{SG}}}\left(\frac{1}{Z q \int_{0}^{w(x)} N_{\mathrm{D}}(y) \mu(y) \mathrm{d} y}\right) \mathrm{d} x .
$$




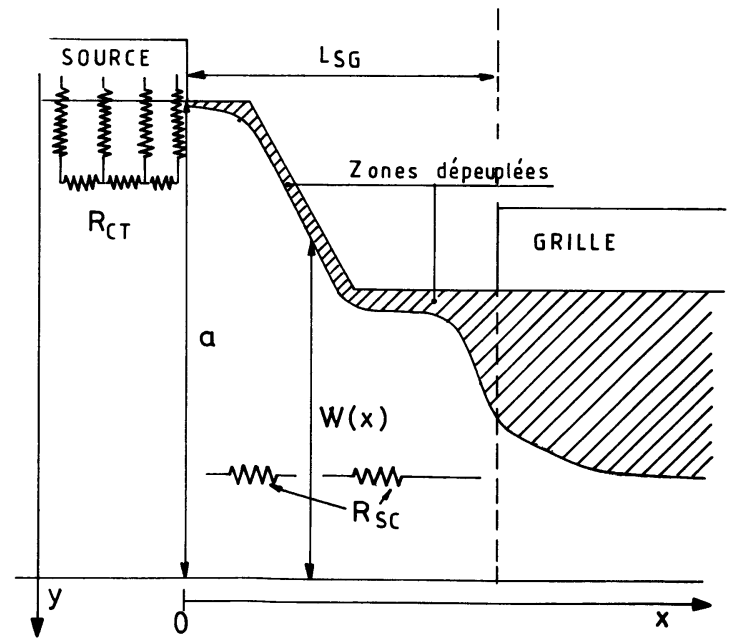

Fig. 5. - Les diverses composantes de la résistance d'accès de source $R_{\mathrm{S}}$ d'un MESFET à grille creusée.

[Decomposition of the source serie resistance for a recessed gate GaAs MESFET.]

Un raisonnement analogue conduit au calcul de la résistance d'accès $R_{\mathrm{D}}$.

ii) La résistance d'accès $R_{\mathrm{G}}$.

La résistance de grille $R_{\mathrm{G}}$ est calculée, en tenant compte de la distribution du courant le long de l'électrode, par la relation suivante [1] :

$$
R_{\mathrm{G}}=\left[\frac{1}{3} \frac{\rho_{\mathrm{g}}}{L} \frac{Z}{H_{\mathrm{G}}}\right] \frac{1}{\left[N_{\mathrm{K}}\right]^{2}}
$$

où $\rho_{\mathrm{g}}, H_{\mathrm{G}}, N_{\mathrm{K}}$ sont définies au paragraphe 2.2.1.

iii) La transconductance intrinsèque $g_{\mathbf{m}_{0}}$.

La transconductance extrinsèque ou pente $g_{\mathrm{m}}$ est calculée dans le modèle en effectuant un incrément $\Delta V_{\mathrm{GS}}$ égal à :

$$
\Delta V_{\mathrm{GS}}=\left(V_{\mathrm{GS}}-V_{\mathrm{T}}\right) / 50
$$

et en déterminant la variation correspondante $\Delta I_{\mathrm{DS}}$ du courant. On obtient alors :

$$
g_{\mathrm{m}}=\left.\frac{\Delta I_{\mathrm{DS}}}{\Delta V_{\mathrm{GS}}}\right|_{V_{\mathrm{DS}}=\mathrm{Cte}}
$$

iv) La conductance de sortie $g_{\mathrm{d}_{0}}$ (intrinsèque).

Le principe du calcul est identique à celui de la transconductance :

$$
g_{\mathrm{d}}=\left.\frac{\Delta I_{\mathrm{DS}}}{\Delta V_{\mathrm{GS}}}\right|_{V_{\mathrm{GS}}=\mathrm{Cte}}
$$

et

$$
\begin{aligned}
g_{\mathrm{d}_{0}} & =\frac{g_{\mathrm{d}}}{1-R_{\mathrm{S}} g_{\mathrm{m}}-\left(R_{\mathrm{S}}+R_{\mathrm{D}}\right) g_{\mathrm{d}}} \\
g_{\mathrm{m}_{\mathrm{o}}} & =\frac{g_{\mathrm{m}}}{1-R_{\mathrm{S}} g_{\mathrm{m}}-\left(R_{\mathrm{S}}+R_{\mathrm{D}}\right) g_{\mathrm{d}}}
\end{aligned}
$$

où $g_{\mathrm{d}_{0}}$ et $g_{\mathrm{m}_{0}}$ sont les valeurs intrinsèques qui ne sont égales aux valeurs extrinsèques que lorsque $R_{\mathrm{S}}=$ $R_{\mathrm{D}}=0$.

Ces relations sont établies en utilisant des méthodes classiques d'analyse des circuits [9].

En régime saturé, nous tenons compte d'une injection de porteurs dans le substrat afin de calculer la conductance de sortie dynamique.

$$
g_{\mathrm{d}_{0}}^{\prime}=g_{\mathrm{d}_{0}}+\frac{1}{K\left(L-L_{1}\right)}
$$

où $K$ est un facteur d'injection empirique sensiblement constant pour tous les composants et égal à $10^{9} \Omega / \mathrm{m}[10]$.

\section{v) La résistance et la capacité d'entrée.}

On représente la région ohmique du canal sous la grille par un ensemble de cellules $R_{\mathrm{j}} C_{\mathrm{j}}$, assimilables à une ligne répartie non uniforme. Cette ligne est chargée par la capacité de la région saturée $C_{\mathrm{ch}}$ (voir Fig. 6).

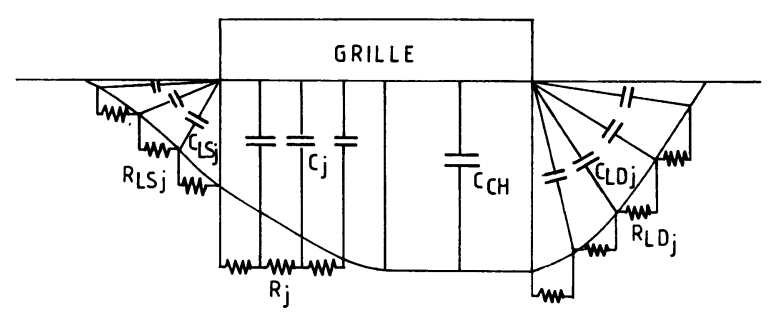

Fig. 6. - Impédance d'entrée du MESFET décrite par une ligne de transmission non uniforme.

[Input impedance of the MESFET equivalent to a nonuniform $R-C$ transmission line.]

Il convient de plus dans ce canal de tenir compte des extensions latérales de la zone de charge d'espace au moyen des capacités $C_{\mathrm{LS}_{\mathrm{j}}} \ldots C_{\mathrm{LD}_{\mathrm{j}}}$ et des résistances $R_{\mathrm{LS}_{j}} \ldots R_{\mathrm{LD}_{j}} \ldots$ qui constituent autant de lignes $R-C$ non uniformes en cascade avec la ligne précédente. L'impédance d'entrée de la ligne constituée par la mise en cascade de l'ensemble des cellules de la figure 6 se synthétise selon un réseau $R_{\mathrm{i}} C_{\mathrm{i}}$ qui représente l'impédance d'entrée du composant (voir Fig. 2). Cette impédance se calcule à partir des expressions classiques des lignes [11].

vi) La capacité grille-drain.

Nous calculons la variation des charges électriques $Q$ (charges stockées dans les zones dépeuplées en porteurs) qui est consécutive à une fluctuation $\Delta V_{\mathrm{DG}}$ du potentiel grill-drain. On obtient donc :

$$
C_{\mathrm{GD}}=\left.\frac{\Delta Q}{\Delta V_{\mathrm{DG}}}\right|_{V_{\mathrm{GS}}=\mathrm{Cte}} .
$$


vii) Le temps de transit.

Le temps de transit des porteurs sous la grille est déterminé par :

$$
\tau_{0}=\int_{0}^{L} \frac{1}{V(E)} \mathrm{d} x
$$

soit en remplaçant $V(E)$ par son expression (4), on peut écrire :

$$
\tau_{0}=\tau_{1}+\tau_{2}
$$

avec

$$
\tau_{1}=\frac{1}{\mu_{0}} \int_{0}^{L_{1}}\left(\frac{1}{E}+\frac{1}{E_{0}}\right) \mathrm{d} x
$$

et

$$
\tau_{2}=\frac{\left(L-L_{1}\right)}{V_{\text {sat }}}
$$

2.3.3 Performances dynamiques. - On rend compte des performances dynamiques du composant en régime de petits signaux essentiellement au moyen des deux quantités suivantes [11] :

- la fréquence de coupure du gain en courant

$$
\begin{aligned}
f_{\mathrm{T}}= & \frac{g_{\mathrm{m}_{\mathrm{o}}}}{2 \pi\left(C_{\mathrm{i}}+C_{\mathrm{gsim}}\right)} \times \\
& \times\left[1+2\left(C_{\mathrm{GD}}+C_{\mathrm{gdim}}\right) /\left(C_{\mathrm{i}}+C_{\mathrm{gsim}}\right)\right]^{-1 / 2}
\end{aligned}
$$

où $C_{\mathrm{gsim}}$ et $C_{\mathrm{gdim}}$ sont les capacités intermetallisations [1], grille-source et grille-drain respectivement.

- La fréquence maximale d'oscillation intrinsèque

$$
f_{\max }=\frac{f_{\mathrm{T}}}{2} \sqrt{\frac{1}{g_{\mathrm{d}_{0}} \cdot R_{\mathrm{i}}}} .
$$

\section{Résultats obtenus. Comparaison avec des valeurs mesurées.}

3.1 Présentation des travaux. - Le simulateur SIMTEC que nous venons de décrire permet de par sa souplesse d'utilisation un grand nombre d'applications. Par exemple, il peut servir à mettre en évidence l'influence du profil de dopage sur les propriétés électriques d'un MESFET. Ainsi, nous allons comparer les résultats donnés par la simulation, et concernant notamment le courant et la pente à des mesures effectuées sur des transistors implantés GaAs.

Quatre séries de transistors, ayant des profils de dopage différents, ont été fabriquées. Pour chaque série, on a réalisé deux implantations successives de Silicium à travers une couche de nitrure épaisse de $500 \AA$, dans un substrat non compensé de type "L.E.C. " (Liquid Encapsulated Czochralski).

La première implantation est effectuée avec une dose de $2 \mathrm{E} 13 \mathrm{~cm}^{-2}$ pour une énergie de $60 \mathrm{keV}$. Elle permet de créer une couche fortement dopée en sur- face $\left(N_{\mathrm{D}}=3 \mathrm{E} 18 \mathrm{~cm}^{-3}\right)$ nécessaire pour l'établissement de bons contacts ohmiques et la diminution des résistances d'accès. Elle est suivie d'une seconde implantation servant à la réalisation de la couche active $\mathrm{N}$ du transistor. Un recuit thermique $\left(820^{\circ} \mathrm{C}\right.$ pendant $15 \mathrm{~min}$ ) permet de "réactiver » les impuretés dopantes et de " guérir " les défauts provoqués dans le réseau par les implantations. Pendant cette opération, on recouvre l'échantillon par un encapsulant $\left(\mathrm{Si}_{3} \mathrm{~N}_{4}\right)$ pour éviter toute évaporation de l'arsenic.

Les paramètres de la couche $\mathrm{N}$ diffèrent selon les séries et sont regroupés sur le tableau I. Pour chaque implantation, la répartition des impuretés ionisées est supposée être une "gaussienne " conformément à la théorie « LSS » [12]. On aurait pu rechercher une répartition plus exacte tenant compte de moments d'ordre supérieur [12] mais cela ne paraît pas être nécessaire pour les couches GaAs implantées Silicium où la comparaison entre le profil LSS et le profil exact obtenu par sonde ionique fait apparaître une concordance excellente [13]. Le profil correspondant à chaque implantation est donné par :

$$
N_{\mathrm{D}}(y)=\frac{\text { Dose }}{\sqrt{2 \pi} \Delta R_{\mathrm{P}}} \exp \left[-\frac{\left(y-R_{\mathrm{P}}\right)^{2}}{2 \Delta R_{\mathrm{P}}^{2}}\right]
$$

$R_{\mathrm{P}}$ et $\Delta R_{\mathrm{P}}$ sont respectivement le parcours projeté (moment d'ordre 1) et l'écart type (moment d'ordre 2) de cette répartition gaussienne. Leurs valeurs sont déduites des tables de Gibbons [14] :

Tableau I. - Paramètres de la $2^{\mathrm{e}}$ implantation des transistors réalisés. $\Delta R_{\mathrm{P}}$ et $R_{\mathrm{P}}$ sont déterminés d'après la référence [14].

[Parameters of the second implantation of the devices.]

\begin{tabular}{ccccl}
\hline Série & $\begin{array}{c}\text { Energie } \\
\mathrm{keV}\end{array}$ & $\begin{array}{c}\text { Dose } \\
\mathrm{cm}^{-2}\end{array}$ & $\begin{array}{c}R_{\mathrm{P}} \\
\mu \mathrm{m}\end{array}$ & $\begin{array}{c}\Delta R_{\mathrm{P}} \\
\mu \mathrm{m}\end{array}$ \\
\hline 1 & 250 & $6 \mathrm{E} 12$ & 0,169 & 0,085 \\
2 & 210 & $6 \mathrm{E} 12$ & 0,133 & 0,078 \\
3 & 150 & $6 \mathrm{E} 12$ & 0,079 & 0,06 \\
4 & 120 & $6 \mathrm{E} 12$ & 0,052 & 0,0465 \\
\hline
\end{tabular}

Les profils de dopage théoriques correspondant à chacune des 4 séries sont représentés sur la figure 8.

Les paramètres électriques et géométriques (Fig. 7) communs à toutes les séries sont :

$$
\begin{gathered}
V_{\mathrm{bi}}=0,75 \mathrm{~V}, \quad L=1 \mu \mathrm{m}, \quad Z=150 \mu \mathrm{m}, \\
L_{\mathrm{SG}}=L_{\mathrm{GD}}=2 \mu \mathrm{m} .
\end{gathered}
$$

La profondeur $R$ du « recess » est variable et permet d'ajuster, lors de la réalisation, la tension de pincement $V_{\mathrm{P}}$ du composant.

Notons que les contacts ohmiques sont réalisés par le dépôt d'un alliage $(\mathrm{Au}(88 \%)-\mathrm{Ge}(12 \%)+$ 


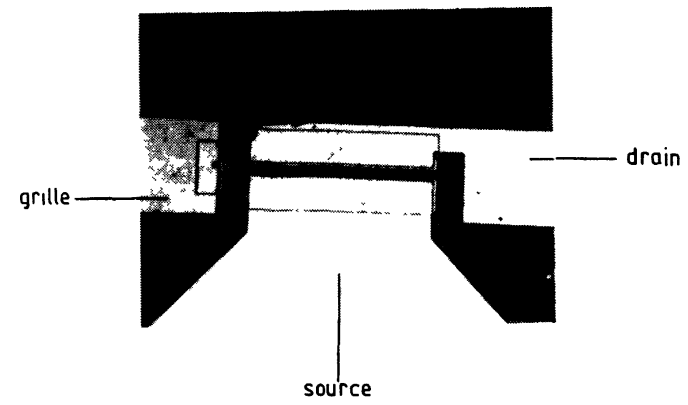

Fig. 7. - Photographie de l'un des transistors réalisé $(Z=150 \mu \mathrm{m})$ pour cette étude.

[Microphotograph of one of the devices to be investigated.]

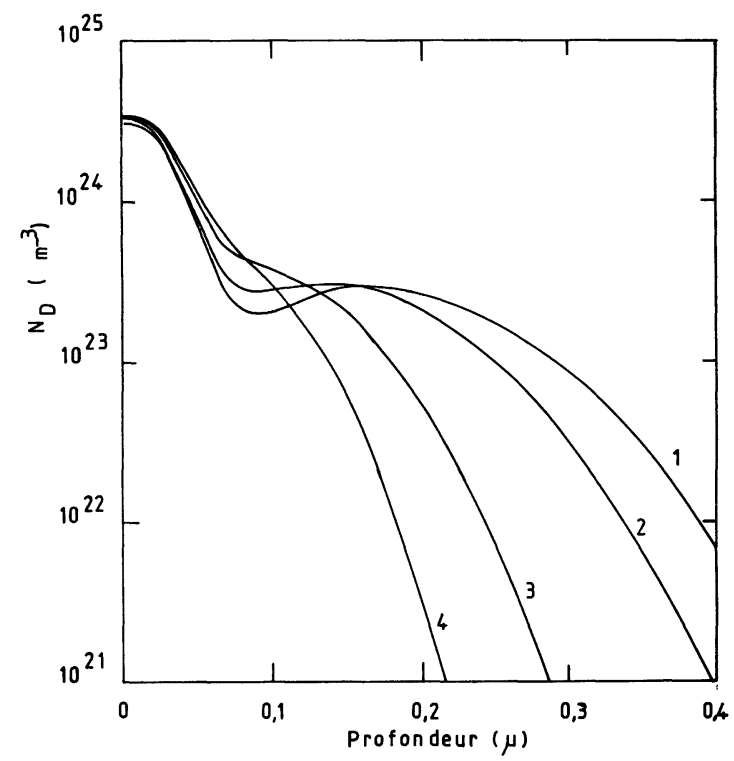

Fig. 8. - Profils de dopages théoriques (LSS) des quatre séries étudiées. Les numéros des courbes correspondent à ceux du texte et à ceux des figures 10 à 17 .

[Implanted doping profiles of each of the four wafers theoretically and experimentally investigated in this paper. The numbers 1 to 4 associated with each wafer will be subsequently used in the text and in figures 10 to 17.]

$\mathrm{Ni)}$ et qu'ils présentent une résistance spécifique de l'ordre de $\rho_{\mathrm{c}}=1 \times 10^{-6} . \mathrm{cm}^{2} \Omega$.

Enfin, la grille (d'une épaisseur de $6000 \AA$ ) est réalisée à partir d'évaporations successives de Ti-Pt-Au par une technique de « lift-off ».

3.2 Comparaison des résultats De la SimulaTION AVEC DES MESURES RÉALISÉES SUR UN DES COMPOSANTS. - Ce composant est pris dans la série 1 $(250 \mathrm{keV})$ et présente un « recess » d'environ $1500 \AA$ qui correspond à une tension grille de seuil égale à $-3,38 \mathrm{~V}$.

Les résistances d'accès calculées par SIMTEC au moyen des expressions (13) et (14) donnent $R_{\mathbf{S}}+$ $R_{\mathrm{D}}=6,8 \Omega$. Ceci est en bon accord avec la valeur mesurée par des techniques classiques [1] qui est égale à $6,4 \Omega$.

La figure 9 permet de comparer les caractéristiques de sortie $I_{\mathrm{DS}}=f\left(V_{\mathrm{DS}}\right)$, mesurées et calculées, pour plusieurs valeurs de $V_{\mathrm{GS}}$.

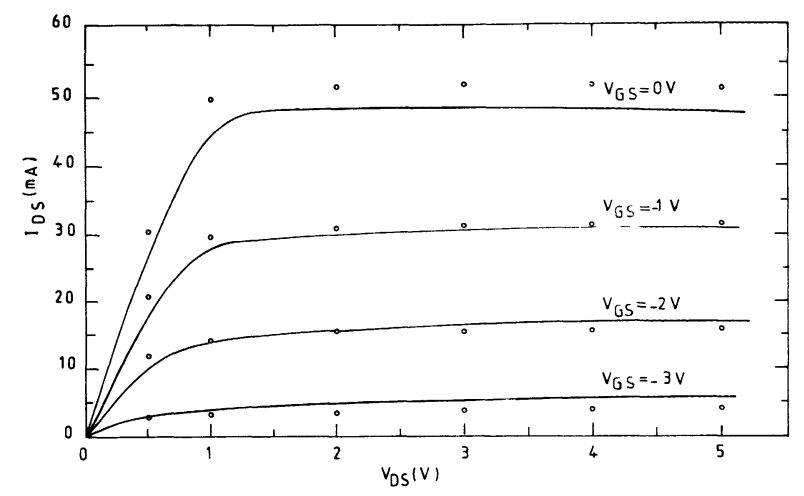

Fig. 9. - Caractéristiques statiques de sortie (composant de la série $1, V_{\mathrm{T}}=-3,38 \mathrm{~V}$ ), mesurées (traits pleins); calculées avec le simulateur SIMTEC (cercle) $\bigcirc$.

[Output current-voltage characteristics of a device from the wafer number $1\left(V_{\mathrm{T}}=-3.38 \mathrm{~V}\right)$. Measured (solid lines); computed with SIMTEC (circles).]

L'accord est excellent sur l'ensemble. On remarque toutefois pour $V_{\mathrm{GS}}=0$ que le courant simulé est un peu plus important (environ $5 \%$ ) que celui mesuré. Ce léger écart provient de l'ambiguité sur la profondeur exacte du recess du fait que le profil de dopage introduit comme donnée d'entrée est un profil théorique (LSS). Or, le profil réel peut être sensiblement différent si le taux d'activation des impuretés s'écarte de $100 \%$ ou si une redistribution intervient. Des résultats plus précis peuvent être obtenus sur des composants où l'on connaît exactement le profil de dopage par caractérisation SIMS, $C-V$, etc...

\subsection{COMPARAISON CALCULS-MESURES CONCERNANT LA} PENTE DU TRANSISTOR DANS LES 4 SÉRIES. - Nous avons porté sur la figure 10 les valeurs de $g_{\mathrm{m}}$ normalisées par rapport à la pente du transistor ayant le plus faible $g_{\mathrm{m}}$ (série $3, V_{\mathrm{P}}: 1,3 \mathrm{~V}$ ) en fonction de la tension de pincement $V_{\mathrm{P}}$ du composant. Les résultats de notre simulation s'accordent très bien avec les résultats expérimentaux. Seule la série 4 paraît échapper à la comparaison. Il faut toutefois souligner pour cette série, que la pente du profil de dopage correspondant (Fig. 8) est très raide. On montre dans ce cas que l'approximation abrupte $(\$ 2.3)$ n'est plus justifiée [7]. Ceci explique que les valeurs mesurées de la pente soient plus faibles que celles prévues par la simulation simplifiée.

Au vu de la figure 10, il apparaît que plus l'énergie d'implantation augmente et plus la dispersion de la pente en fonction de la tension de pincement diminue. 


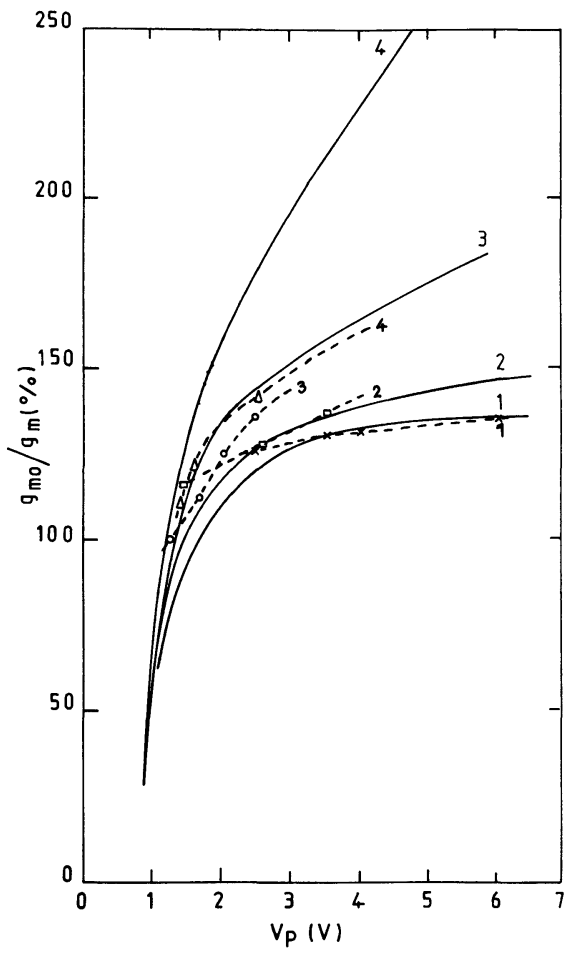

Fig. 10. - Comparaison des variations de la pente normalisée $g_{\mathrm{m}_{0}} / g_{\mathrm{m}_{0}}(1)$ en fonction de la tension de pincement $V_{\mathrm{P}}$ $\left(g_{m_{0}}(1)\right.$ représente la pente du transistor de la série 3 possédant une tension $V_{\mathrm{p}}$ égale à $1,3 \mathrm{~V}$ ) calculées avec le simulateur SIMTEC (traits pleins). Séries 1, 2, 3 et 4 ; mesures série 1 (croix); mesures série 2 (carrés); mesures série 3 (cercles); mesures série 4 (triangles).

[Variations of the normalized transconductances $\left(g_{\mathrm{m}_{0}}(1)\right.$ from wafer $\left.3, V_{\mathrm{P}}=1.3 \mathrm{~V}\right)$. Computed with SIMTEC (solid lines). Wafers : 1, 2, 3 and 4; measured wafer 1 (cross); measured wafer 2 (squares); measured wafer 3 (circles); measured wafer 4 (triangles).]

Par contre, les valeurs élevées de $g_{\mathrm{m}_{0}}$ (qui peuvent être intéressantes pour des applications faible bruit) sont obtenues pour les implantations à plus faible énergie.

Les résultats expérimentaux confirment tout à fait ces remarques.

\section{Influence du profil de dopage sur les performances d'un MESFET.}

Pour les quatre séries de transistors précédentes, nous présentons, à titre d'illustration des possibilités du simulateur, l'évolution théorique des principaux éléments du schéma équivalent : $g_{\mathrm{m}_{0}}, \tau_{0}, g_{\mathrm{d}_{0}}, R_{\mathrm{i}}, C_{\mathrm{i}}, C_{g_{\mathrm{d}}}$, $f_{\mathrm{T}}$ et $f_{\mathrm{MAX}}$ en fonction de la tension de pincement du composant (Figs. 10 à 17).

Ceci revient en pratique à étudier l'influence de la profondeur $\mathrm{du}$ " recess » sur les propriétés électriques du composant.

Toutes ces courbes ont été obtenues pour $V_{\mathrm{GS}}=$ $0 \mathrm{~V}$ et $V_{\mathrm{Ds}}=3 \mathrm{~V}$, c'est-à-dire lorsque le courant drain- source est égal à $I_{\text {Dss. }}$. Ces courbes suscitent un certain nombre de commentaires :

a) La transconductance $g_{\mathrm{m}_{0}}-$ Les résultats obtenus confirment les résultats précédents et mettent en évidence l'intérêt d'une faible énergie d'implantation pour optimiser la pente. En effet, une énergie d'implantation faible entraîne une faible épaisseur de la couche dopée, ce qui correspond à des valeurs de la pente élevées [1].

b) Le temps de transit des porteurs sous la grille $\tau_{0}$. - Au vu de la figure 11, il apparait que le temps de transit dépend assez peu de la tension de pincement du transistor, mais dépend par contre de l'énergie d'implantation. Les valeurs les plus faibles du temps de transit sont obtenues pour les implantations aux plus fortes énergies (séries 1 et 2). Pour ces énergies, la valeur moyenne du dopage de la couche active après creusement de grille est la plus faible. Ainsi la valeur élevée de la mobilité $\mu_{0}$ ainsi obtenue (Rel. (3)) permet de minimiser le temps de transit des porteurs.

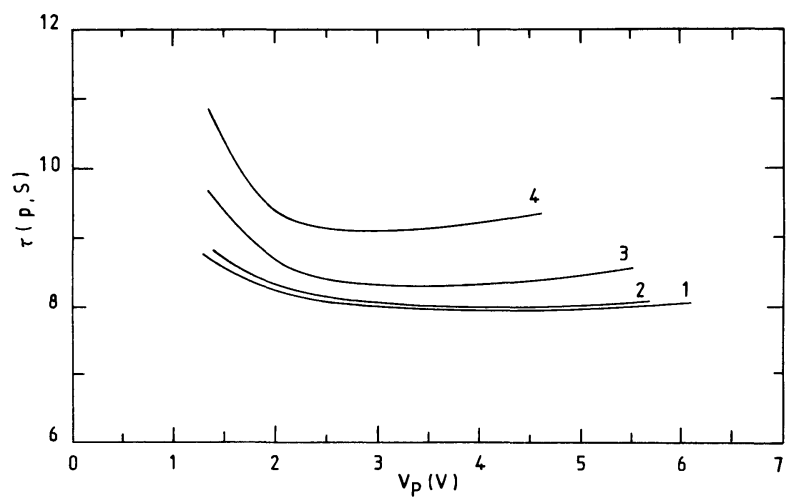

Fig. 11. - Variations théoriques du temps de transit des porteurs sous la grille $\tau_{0}$ en fonction de la tension de pincement pour chacune des quatre séries (voir Tableau I).

[Theoretical variations of transit time under the gate $\tau_{0}$ versus pinch-off voltage for the wafers number 1 to 4 .]

c) La conductance de sortie $g_{\mathrm{d}_{0}}$ - La figure 12 montre l'intérêt de la série 1 lorsqu'on recherche une résistance dynamique drain-source élevée. (Applications en charge active par exemple.) Cela provient du fait que, pour les fortes énergies, le dopage dans le canal conducteur sous la grille, en régime saturé, est inférieur à $10^{22} \mathrm{~m}^{-3}$.

d) La résistance d'entrée $R_{\mathrm{i}}$ - La figure 13 indique

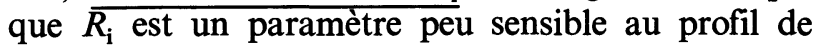
dopage.

e) La capacité d'entrée $C_{\mathrm{i}}$. - On retrouve ici l'intérêt de la série 1 . Une implantation à forte énergie correspond à des faibles valeurs de la capacité d'entrée. En effet, pour ces énergies, le dopage moyen de la 


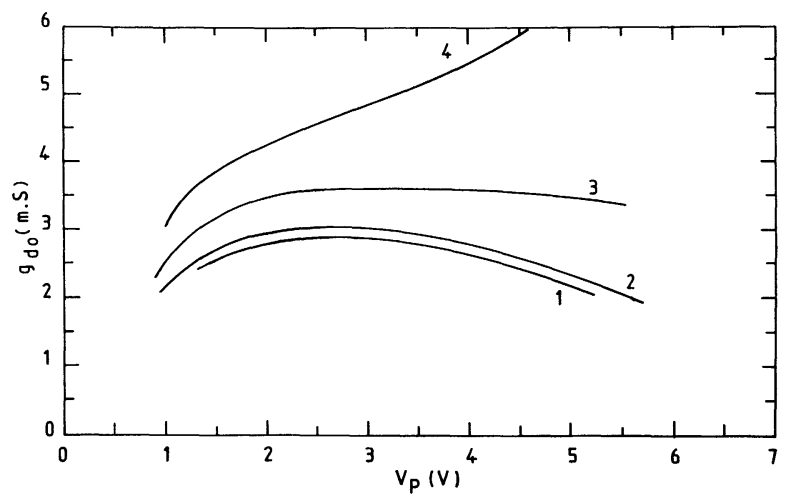

Fig. 12. - Variations théoriques de la conductance de sortie $g_{\mathrm{d}_{\mathrm{o}}}$ en fonction de la tension de pincement pour chacune des quatre séries (voir Tableau I).

[Theoretical variations of output conductance $g_{\mathrm{d}_{0}}$ versus pinch-off voltage for the wafers number 1 to 4 .]

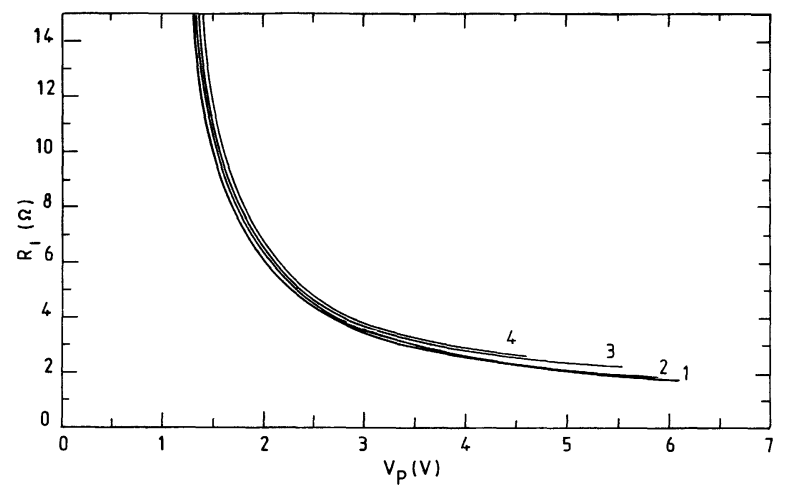

Fig. 13. - Variations théoriques de la résistance d'entrée $R_{\mathrm{i}}$ en fonction de la tension de pincement pour chacune des quatre séries (voir Tableau I).

[Theoretical variations of input resistance $R_{\mathrm{i}}$ versus pinchoff voltage for the wafers number 1 to 4 .]

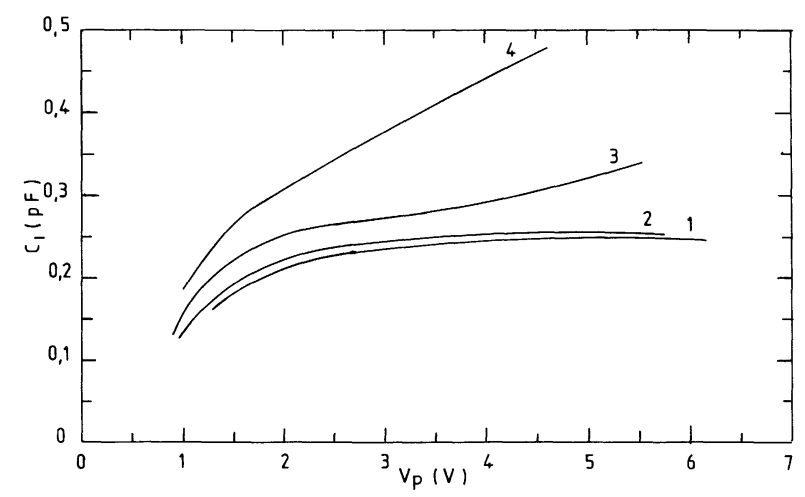

Fig. 14. - Variations théoriques de la capacité d'entrée $C_{\mathrm{i}}$ en fonction de la tension de pincement pour chacune des quatre séries (voir Tableau I).

[Theoretical variations of input capacitance $C_{\mathrm{i}}$ versus pinch-off voltage for the wafers number 1 to 4 .] couche active, après creusement de grille, est faible. Ainsi, l'épaisseur de la zone dépeuplée sous la grille est plus élevée et par suite la capacité d'entrée $C_{\mathbf{i}}$ est minimale. Cette propriété est très intéressante par exemple pour une utilisation en commutation.

f) La capacité entrée-sortie $C_{g_{\mathrm{d}^{-}}}$- La figure 15 fait apparaître, notamment pour les séries 3 et 4 , l'existence d'une tension de pincement idéale (et donc d'une profondeur de « recess » optimum) pour s'assurer d'une bonne isolation entrée-sortie. Les implantations à fortes énergies exigent, pour obtenir une tension de pincement donnée, un creusement ( recess ») plus important, supérieur à $0,1 \mu \mathrm{m}$. Au droit de l'électrode de grille, près de la surface, on se trouve alors dans une région faiblement dopée (Figs. 1 et 8) si bien que l'extension latérale de la zone dépeuplée en direction du drain devient importante. De ce fait, la capacité $C_{g_{\mathrm{d}}}$ (dont une composante est inversement proportionnelle à cette extension) est alors minimale comme on peut le vérifier sur la figure 15 .

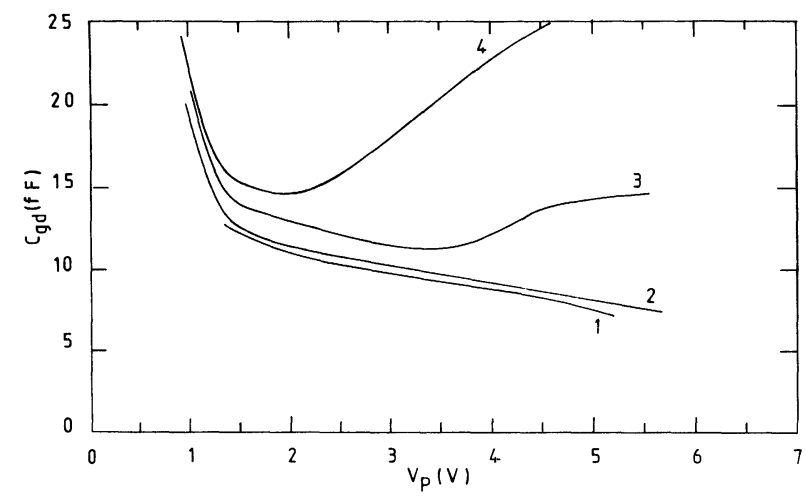

Fig. 15. - Variations théoriques de capacité entrée-sortie $C_{g_{\mathrm{d}}}$ en fonction de la tension de pincement pour chacune des quatre séries (voir Tableau I).

[Theoretical variations of input-output capacitance $C_{g_{\mathbf{d}}}$ versus pinch-off voltage for the wafers number 1 to 4.]

g) La fréquence de coupure du gain en courant. $\mathrm{Au}$ vu de la figure 16 , on peut conclure que $f_{\mathrm{T}}$ (environ $15 \mathrm{GHz}$ ) est pratiquement indépendante du profil de dopage et de la tension de pincement. Remarquons que la valeur de $f_{\mathrm{T}}$ mesurée sur ces transistors reste constante (de l'ordre de $12 \mathrm{GHz}$ ) ce qui confirme l'analyse théorique. Un tel résultat s'explique par l'étude du fonctionnement du composant qui montre que la fréquence de coupure dépend essentiellement de la longueur de grille [1].

h) La fréquence maximale d'oscillation. - Là encore, il apparait sur la figure 17 que les séries 1 et 2 sont les plus intéressantes pour un fonctionnement à fréquence élevée. Ceci s'explique par le fait que ces séries présentent une résistance dynamique élevée 


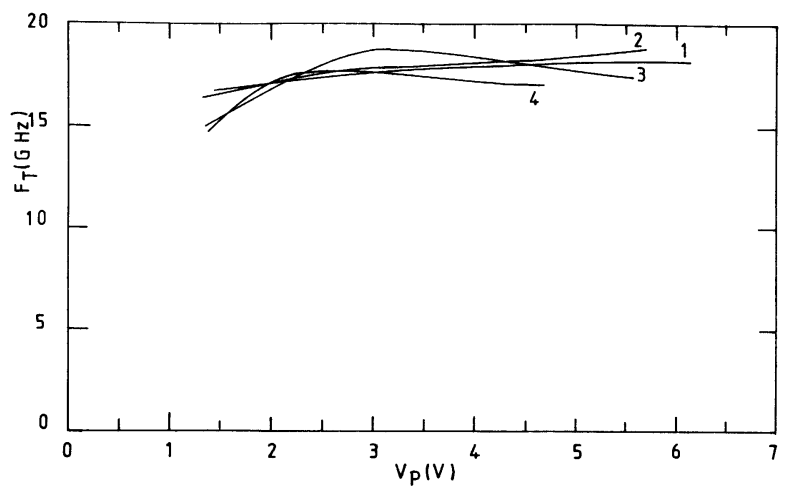

Fig. 16. - Variations théoriques de la fréquence de coupure en courant $f_{\mathrm{T}}$ en fonction de la tension de pincement pour chacune des quatre séries (voir Tableau I).

[Theoretical variations of current out off frequency $f_{\mathrm{T}}$ versus pinch-off voltage for the wafers number 1 to 4 .]

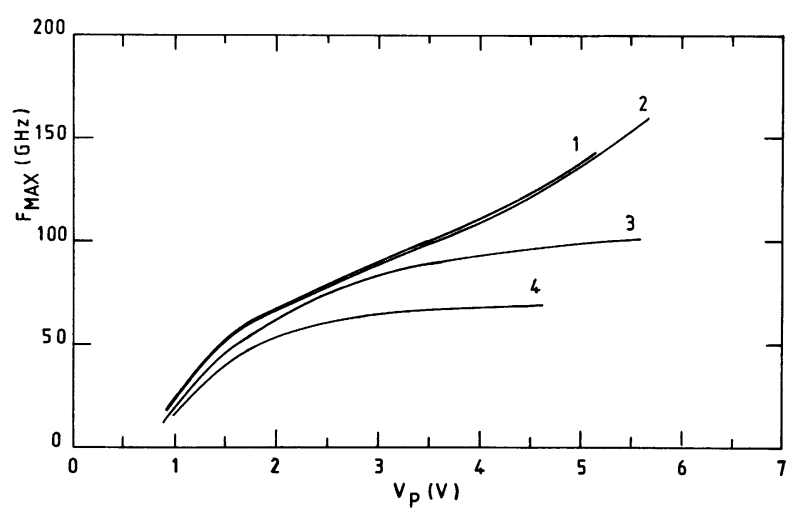

Fig. 17. - Variations théoriques de la fréquence maximale d'oscillation intrinsèque en fonction de la tension de pincement pour chacune des quatre séries (voir Tableau I).

[Theoretical variations of intrinsic maximum frequency of oscillation versus pinch-off voltage for the wafers number 1 to 4.]

(Rel. (25)). On constate aussi que pour améliorer la fréquence maximale d'oscillation intrinsèque, on ne doit pas trop creuser avant de déposer la grille afin de conserver une tension de pincement $V_{\mathrm{P}}$ assez importante.

L'ensemble des figures fait apparaître que la sensibilité de la plupart des paramètres électriques à la tension seuil, donc à la profondeur du " recess", est d'autant plus faible que l'énergie d'implantation est élevée. Cette propriété est très intéressante lorsqu'il est important d'obtenir, en dépit de toutes les dispersions technologiques inévitables, une bonne homogénéité des performances électriques sur plusieurs composants réalisés simultanément dans les circuits intégrés par exemple.

Remarquons cependant qu'une énergie d'implantation élevée permet d'optimiser la fréquence maximale d'oscillation du composant, mais que par contre la pente obtenue est nettement inférieure à celle qu'autoriserait une énergie d'implantation plus faible. Ceci pourrait être néfaste pour les applications à faible bruit par exemple.

\section{Conclusion.}

En conclusion, le logiciel SIMTEC apparaît être un outil parfaitement adapté pour l'optimisation des caractéristiques technologiques des composants en fonction des applications visées. De plus, en raison des temps de calcul très faibles nécessaires $(1 \mathrm{~s}$ sur calculateur type IBM 3080 pour 10 points de polarisation), il permet de simuler des circuits intégrés à faible densité d'intégration et d'optimiser ainsi leurs performances [15].

\section{Remerciements.}

La partie de cette étude réalisée au L.A.A.S. a été soutenue par la D.A.I.I. (Contrat $\mathbf{n}^{\circ}$ 82.35.009.00.790. 75.00). Nous remercions A. Perrichon, Ingénieur C.N.E.T. Lannion, pour les commentaires et les conseils prodigués à l'occasion de cette étude ainsi que U.T. Pham Pham, Ingénieur Thomson-Semiconducteurs (DAG) pour son support concernant la réalisation des transistors.

\section{Annexe I}

CORRECTION THERMIQUE SUR $I_{\mathrm{DS}}$ - Lorsque le composant est traversé par un courant important, il dissipe de la puissance et sa température augmente. Cette variation de température entraîne des changements dans la mobilité à champ nul, la vitesse de saturation des électrons et dans la hauteur de barrière du contact Schottky de grille.

Le courant entre drain et source est donc modifié. Nous proposons les corrections thermiques suivantes :

$$
\begin{array}{ll}
\mu_{0}^{\prime}=\frac{\mu_{0}}{D_{\mathrm{T}}} & \text { avec } \quad D_{\mathrm{T}}=1+\left[\frac{I_{\mathrm{DS}} V_{\mathrm{DS}} R_{\mathrm{TH}}}{T_{0}}\right]^{3 / 2} \\
V_{\text {sat }}^{\prime}=\frac{V_{\text {sat }}}{D_{\mathrm{T}}} &
\end{array}
$$

et

$$
V_{\mathrm{bi}}=V_{\mathrm{bi}}-\alpha\left(I_{\mathrm{DS}} \cdot V_{\mathrm{DS}} \cdot R_{\mathrm{TH}}\right) .
$$

- $T_{0}$ est la température ambiante en Kelvin; $R_{\mathrm{TH}}$ la résistance thermique (de l'ordre de $200 \mathrm{~K} / \mathrm{W}$ pour des transistors de type "Faible bruit »); $\alpha$ un coefficient de l'ordre de $1 \mathrm{mV}$ par degré. Il faut souligner que ces variations thermiques sont en accord avec les résultats publiés par T. W. Hickmott [16]. A partir des valeurs corrigées de $\mu_{0}, V_{\text {sat }}$ et $V_{\text {bi }}$ une ou plusieurs itérations successives permettent de calculer la valeur exacte du courant $I_{\mathrm{DS}}$. 


\section{Bibliographie}

[1] Soares, R., Graffeuil, J., Obregon, J., Applications des transistors à effet de champ en arséniure de gallium (Ed. Eyrolles) 1984.

[2] Higgins, J. A., Modeling the influence of carrier profiles on MESFET characteristics. IEEE Trans $E-D 27$ (1980).

[3] Higgins, J. A., Pattanayak, D. N., A numerical approach to Modeling the ultrashort-gate MESFET. IEEE Trans E-D 29 (1982).

[4] Golio, J. M., Trew, R. J., Profile studies of IonImplanted MESFET's. IEEE MTT (1984).

[5] SzE, S. M., Physics of semiconductor devices (Ed. John Wiley and Sons) 1981, p. 29.

[6] Graffeuil, J., Rossel, P., Semiempirical expression for direct transconductance and equivalent saturated velocity in short-length MESFET's IEE Proc. 129 (1982).

[7] A paraître.

[8] Grebene, A. B., Ghandi, S. K., General theory for pinched operation of the junction gate FET. Solid State Electron. 12 (1969).
[9] VAN DER ZIEL, A., ERo, J. W., Thermal noise in Field Effect Transistors. Proc. on IRE, Août 1962, p. 1808-1812.

[10] Graffeuil, J., Rossel, F., Azizi, C., Influence of Field Dependent Mobility and Buffer Isolation Resistance on Hight Frequency performances of GaAs MESFET's. I.E.D.M. Washington, Dec. 1977.

[11] Combes, E. F., Graffeuil, J., Sautereau, J. F., Composants, dispositifs et circuits actifs en micro-ondes (Ed. Dunod) 1985.

[12] RysSEL, H., Range distributions dans Ion implantation techniques (Ed. Springer-Verlag) 1982, p. 177.

[13] Pavlidis, D., Communication personnelle.

[14] Gibbons, J. F., Johnson, W. S., Mybère, S. W., Projected Range Statistics, Semiconductors and related materials.

[15] A paraître.

[16] HickmotT, T. W., Temperature dependence of FET properties for Cr. Doped and LEC Semi-Insulating GaAs Substrates. IEEE Trans. E.D. 31 (1984). 